\title{
Supporting Information: \\ Resonance Energy Transfer Enables Efficient Planar Heterojunction Organic Solar Cells
}

\author{
Obadiah G. Reid ${ }^{\dagger^{\prime}}$ and Garry Rumbles ${ }^{*}, \dot{\dagger}, \dot{t}$ \\ †Chemistry and Nanoscience Center, National Renewable Energy Laboratory, 15013 \\ Denver West Parkway, Golden, CO 80401, United States \\ $\ddagger$ Department of Chemistry and Biochemistry, University of Colorado at Boulder, \\ Boulder, CO 80309, United States. \\ 'Renewable and Sustainable Energy Institute, University of Colorado at Boulder, \\ Boulder, CO 80309, United States. \\ *Corresponding author: \\ garry.rumbles@nrel.gov
}




\section{Example spatial distributions from the $k M C$ model:}
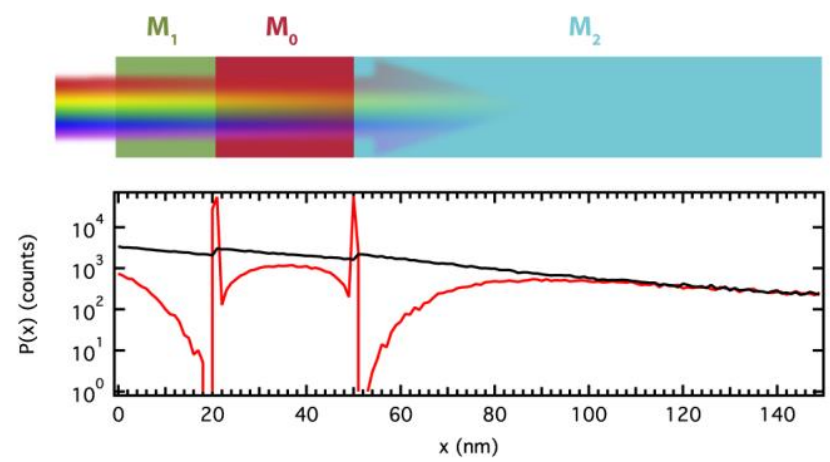

Figure S1) The initial (black) and final (red) distribution of excitons in an example MC simulation for the structure shown above with AM1.5 illumination incident from the left.

Figure S1 shows the spatial distribution of excitons at the beginning (black trace) and end (red trace) of a typical kinetic Monte-Carlo (kMC) simulation for the structure shown in Figure 1 of the main text. The upper panel shows the position and thicknesses of each material layer, and the direction from which AM1.5 illumination is incident. The spikes in the final probability distribution within $\pm 1 \mathrm{~nm}$ of each interface correspond to $\mathrm{kMC}$ trajectories that end with exciton dissociation. Throughout the remainder of the simulation domain the probability amplitude corresponds to $\mathrm{kMC}$ trajectories that end with radiative or non-radiative decay of the exciton. In this case $L_{D}$ was set to $20 \mathrm{~nm}$ in the central layer $\left(\mathrm{M}_{0}\right)$, and $5 \mathrm{~nm}$ elsewhere. The efficacy of long-range energy transfer is evident in the exciton depletion zones on left and right sides of the stack: the critical transfer distance where at least $50 \%$ of the initial exciton density is depleted at the end of the simulation is $30 \mathrm{~nm}$ from the interface with the acceptor $\left(\mathbf{M}_{0}\right)$ in both $\mathbf{M}_{1}$ and $\mathbf{M}_{2}$. In each case the Forster radius for transfer to $M_{0}$ was $\sim 6 \mathrm{~nm}$. 


\section{Simulated Current Density Contour Plots:}
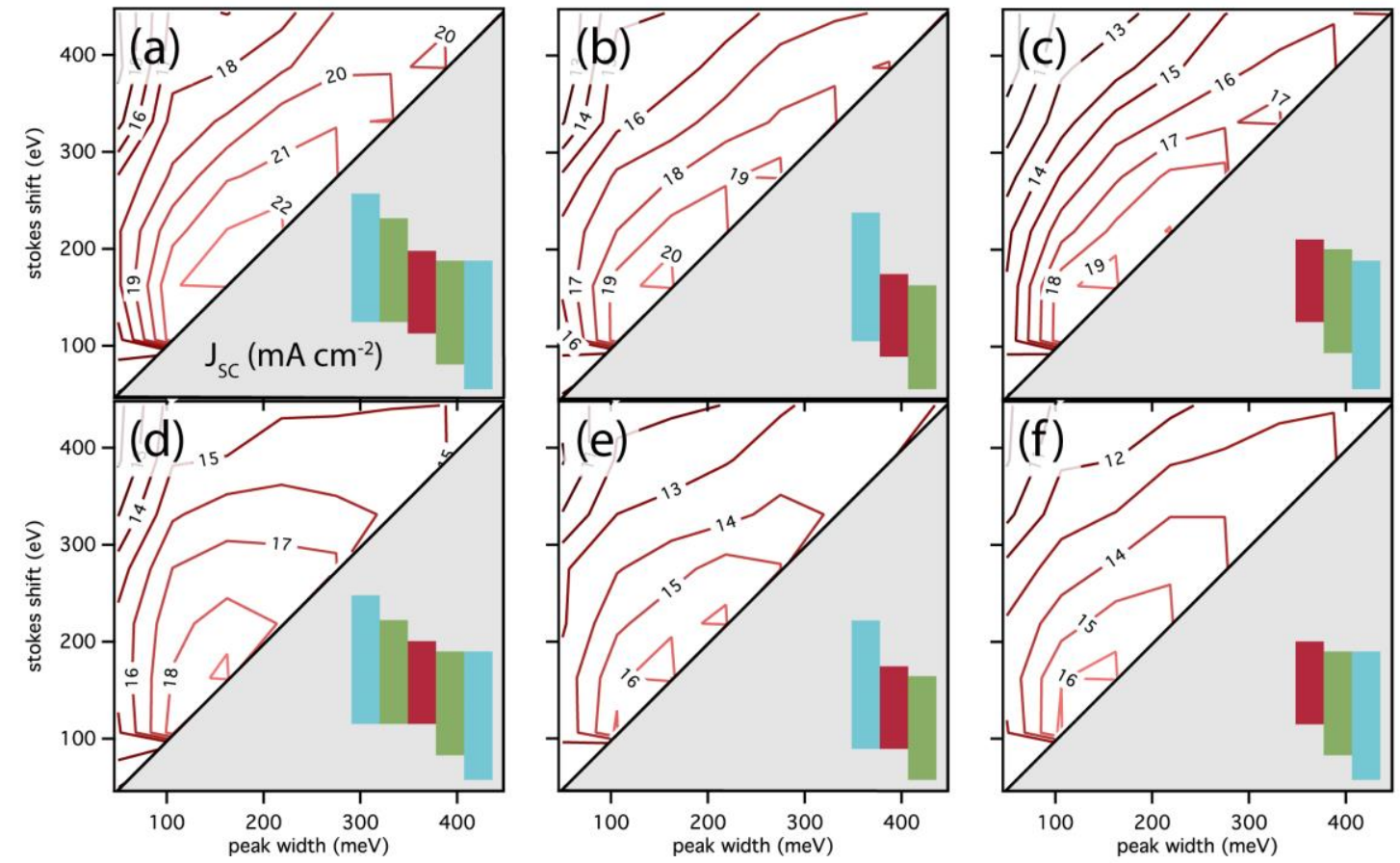

Figure S2) Current density contour plots used to generate the PCE plots in the main text. Current density is given in $\mathrm{mA} / \mathrm{cm}^{2}$ under AM1.5 illumination, assuming $100 \%$ charge collection efficiency. The inset structures show the device configuration for which each contour plot is calculated. The axes on each plot are the stokes-shift (vertical) and Gaussian peak width (horizontal) of the lowest-energy material in the stack. The lowest energy absorption maximum was set at $1.5 \mathrm{eV}$ in all cases. The top row (a-c) shows the current density for optimized configurations with two charge-separating interfaces, while the bottom row (d-f) shows configurations with one charge-separating interface. 


\section{Effect of $S_{2}$ energy on predicted efficiency and optimal spectra:}

AM1.5 PCE (\%)

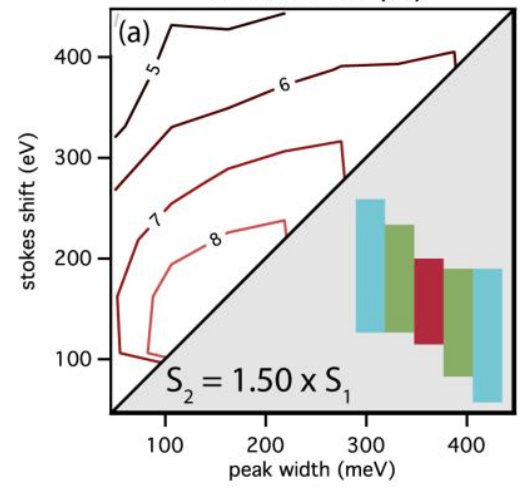

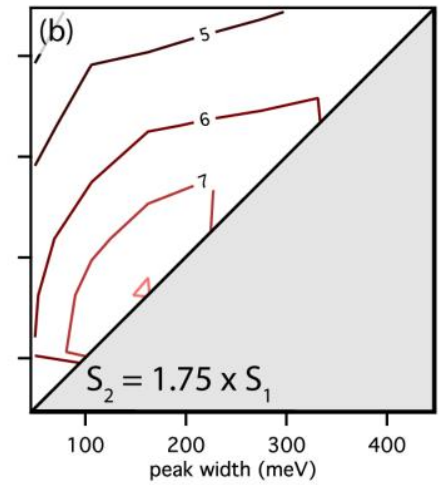

Figure S3) Contour plots of power conversion efficiency calculated for the inset structure in (a) as a function of the second absorption transition energy relative to the first transition in all materials: $1.5 x E_{1}(a), 1.75 x E_{1}(b)$, and $2 x E_{1}(c)$. Increasing the $S_{2}$ energy effectively decreases the oscillator strength available for absorption of the solar spectrum, and results in a concomitant decrease in predicted power conversion efficiency. 


\section{Measurement of integrated absorption coefficient for P3HT and TiOPC:}

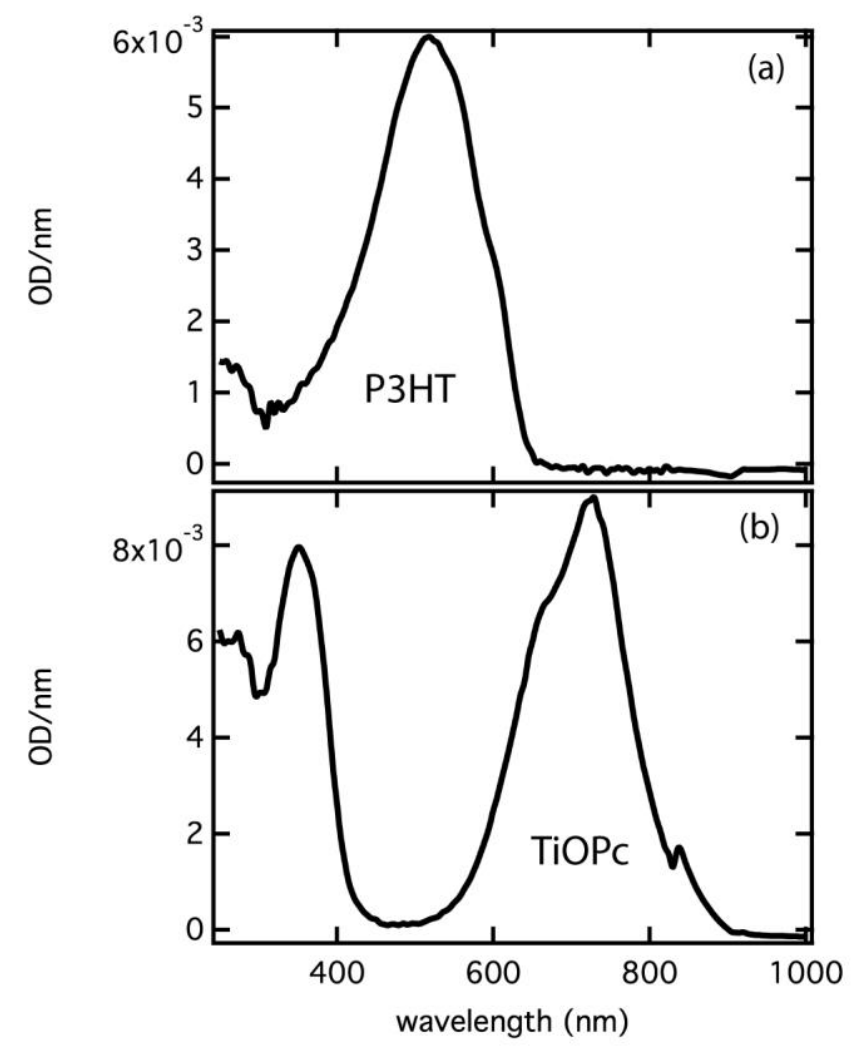

Figure S4) Measured absorption spectra for (a) regioregular-poly(3-hexylthiophene) $(R R-P 3 H T)$, and $(b)$ titanyl phthalocyanine $(T i O P c)$, each normalized for the film thickness as measured by AFM.

Absorption measurements were made on an integrating sphere to account for reflected and scattered light. AFM measurements were performed on scratches made in the same films used for absorbance measurements to determine their thickness. Thickness measurements were made in tapping mode on an Asylum Research MFP-3D microscope. 


\section{Power law scaling of absorption bandwidth:}

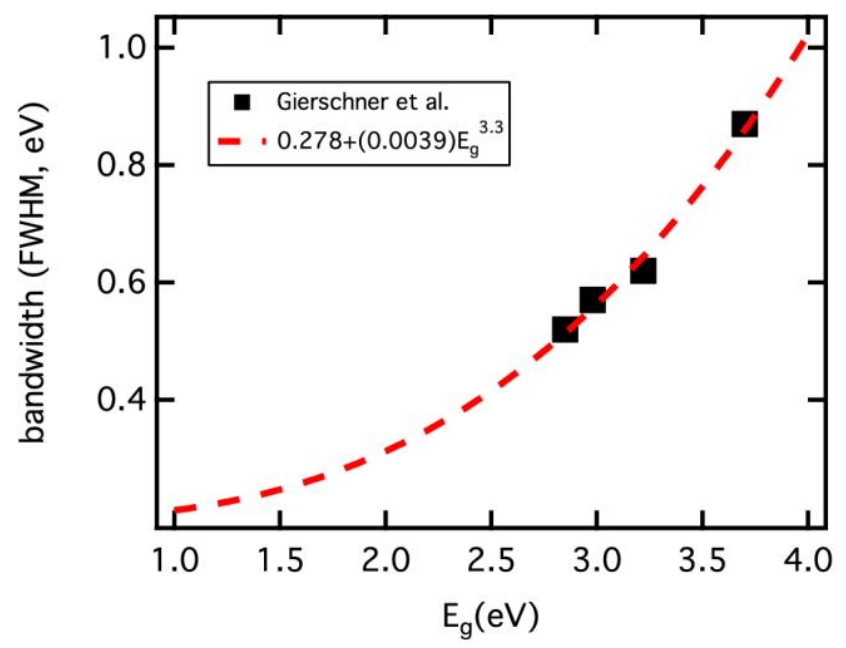

Figure S5) bandwidth (FWHM) vs. energy gap for a series of polyphynelene oligomers taken from Gierschner et al. ${ }^{1}$ (black squares). The red dashed trace shows the power law fit to the data that is used to scale absorption and emission bandwidths with energy in our modeled spectra. The fit parameters are shown in the inset. 


\section{The limit of many-layered structures:}

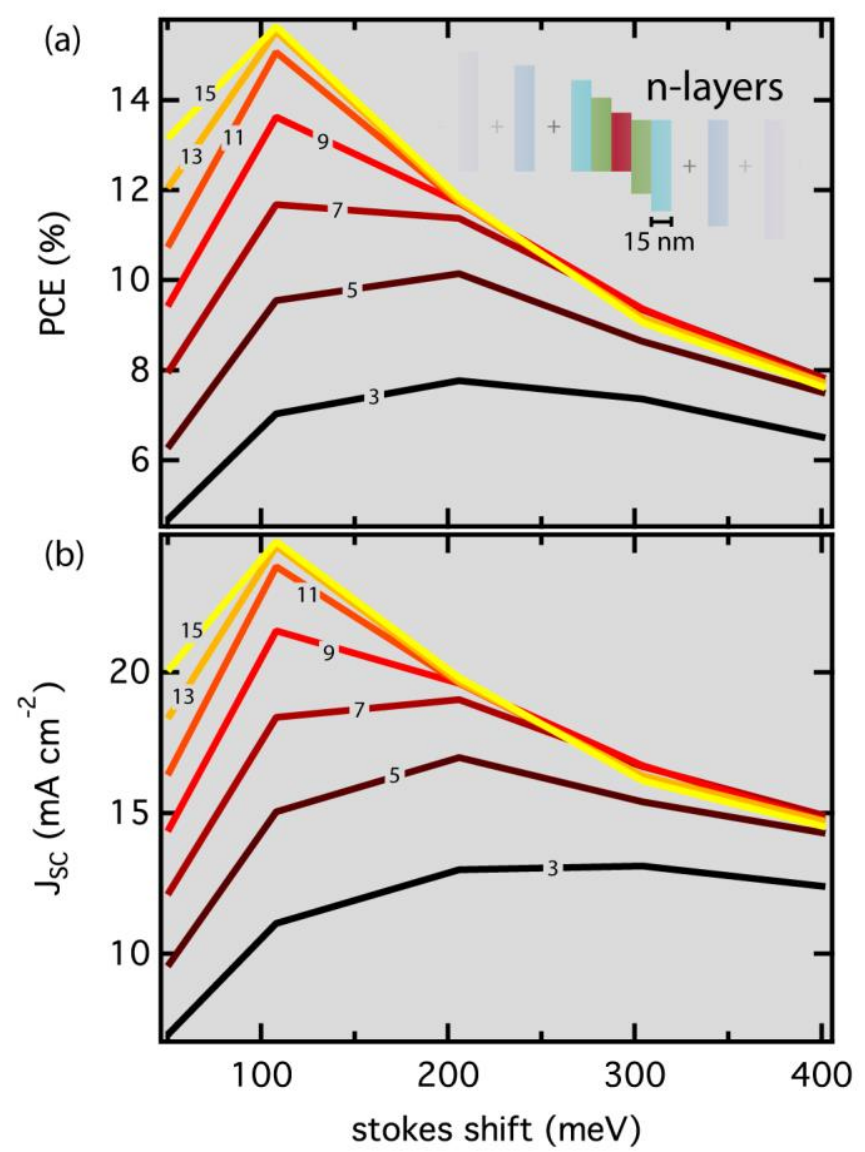

Figure S6) Power conversion efficiency and short-circuit current density vs. stokes shift and absorption bandwidth (bandwidth $=1 / 2$ the stokes shift) for an increasing number of layers in a symmetric stack The number on each solid trace indicates the number of layers used in the calculation. The inset shows the structure being simulated.

In the limit of many layers of material, how efficient can exciton harvesting become? To investigate this we have used the symmetric arrangement shown in $3 \mathrm{~d}$, and varied the number of layers from 3 to 15 . In each case the layer thicknesses were kept constant across the stack, but optimized on a $5 \mathrm{~nm}$ grid for each stack. Likewise, the spectral width was optimized, while keeping the stokes-shift fixed at twice the width. The results are shown in Figure S6 in terms of calculated power conversion efficiency (a) and current density (b).

The photon harvesting efficiency of the stack eventually saturates as the number of layers increases. Both the optimum spectral width and the thickness of each layer decreases with the increasing number of layers. For many layers, the optimum individual layer thickness settles on $\sim 15 \mathrm{~nm}$ reflecting the distance over which exciton transfer is ca. 95\% efficient (see figure 2a). For $100 \mathrm{meV}$ stokes-shift / $50 \mathrm{meV}$ bandwidth, the power conversion efficiency saturates just below $15 \%$.

Clearly, such complex layered structures may be very hard to fabricate. Nevertheless, it is interesting that it appears to be possible to transfer energy across such a large film 
thickness (>100 $\mathrm{nm}$ for the thickest film, 15 layers) with near unit quantum efficiency, and that such high power conversion efficiency can in principle be achieved with this design paradigm.

Figure S7a shows the maximum current density and efficiency predicted as a function of the number of layers in the stack, and the photoluminescence quantum yield of each layer, comparing the results to the maximum current possible for a band gap of $1.4 \mathrm{eV}$; i.e. with the perfect step-function charge pair creation assumed in the Shockley-Queisser (SQ) model. $^{2}$ We see that the current density in the 15 layer structures achieves a value $\left(25.4 \mathrm{~mA} / \mathrm{cm}^{2}\right)$ that is $77 \%$ of the maximum $\left(32.9 \mathrm{~mA} / \mathrm{cm}^{2}\right)$, and that even at the maximum current density, the power conversion efficiency is below $21 \%$. This is a consequence of the rather large energy loss between $\mathrm{E}_{\mathrm{g}}$ and $\mathrm{V}_{\mathrm{OC}}$ that has so far been observed in organic materials: at least $600 \mathrm{meV}$. The detailed balance limit predicts a loss of only $\sim 250 \mathrm{meV}$, and a larger fill factor than the value we have assumed. Thus, unless a carrier multiplication or hot-carrier scheme is employed, no single-junction organic solar cell will exceed $21 \%$ efficiency unless this energy loss can be reduced. Figure S7b shows the absorbance and quantum efficiency spectra that result from the optimized 15-layer structures, and Figure S7c shows the individual absorption spectra for each layer. Note that only 8 spectra are shown because the structure is optically symmetric, as described in the main text. Of particular note, however, is the strong overlap between the spectra of each layer. This situation provides the optimal combination of overlap with the solar spectrum, and efficient energy transport within the multilayer stack. As discussed in the main text, widely separated spectra for each layer are not desirable. 

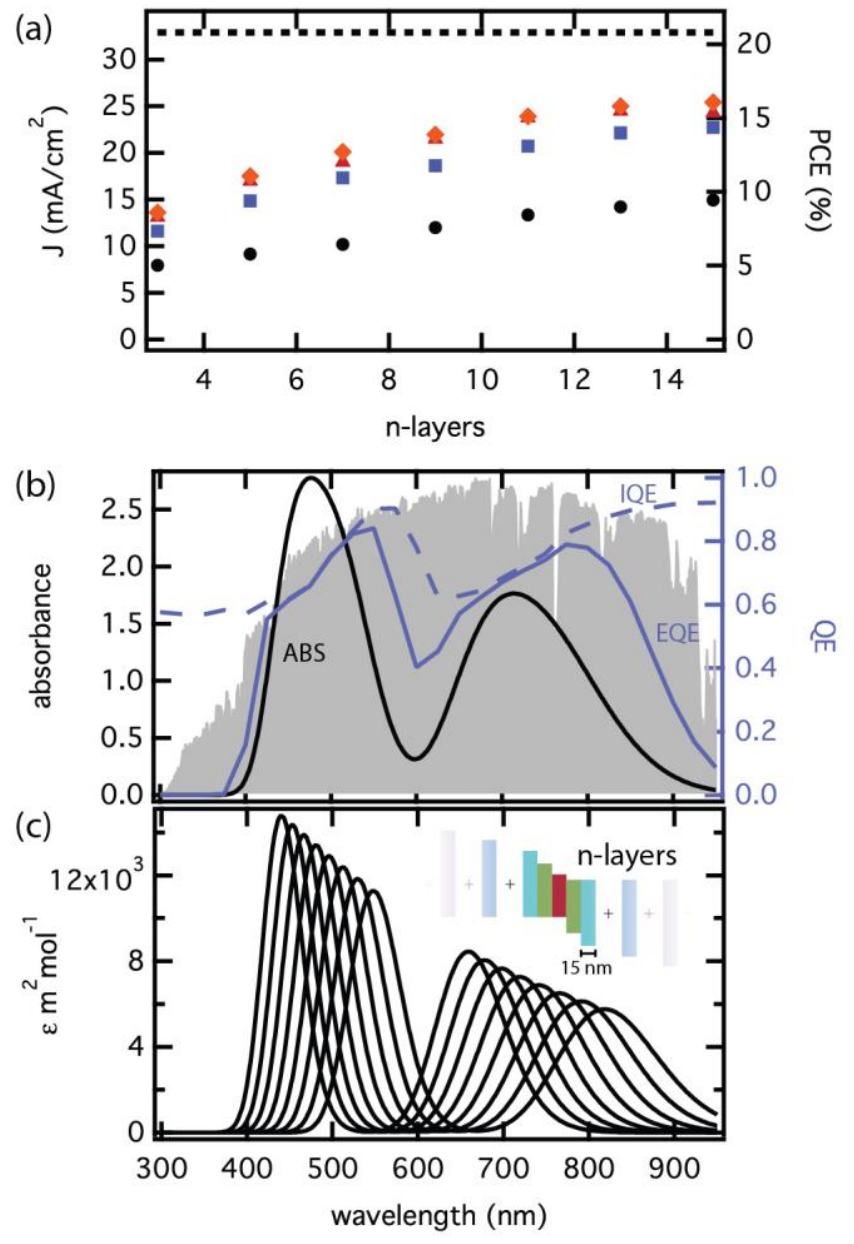

Figure S7) (a) Predicted current density and efficiency as a function of the number of layers in a multilayer energy transfer solar cell with optimized synthetic spectra, constrained such that the stokes-shift is twice the absorption/emission bandwidth in each case. Results are plotted for four different values of the photoluminescence quantum yield of each layer: 10\% (black circles); 50\% (blue squares); $80 \%$ (red triangles) and 100\% (orange diamonds). The dashed black line indicates the maximum current density and efficiency possible given the energy loss $\left(q V_{o c}=\left(E_{g}-\right.\right.$ $600 \mathrm{meV}$ )) and fill factor (0.75) we have assumed. The current density is an absolute maximum for $E_{g}=1.4 \mathrm{eV}$. (b) absorption (black) and quantum efficiency (blue) spectra for the 15-layer stack. The solar irradiance spectrum is plotted in grey. (c) The absorption spectra for each layer in the 15-layer stack. There are only 8 separate spectra because the multilayer is optically symmetric. 


\section{Efficiencies possible with real spectra:}

It is reasonable to question whether the synthetic spectra used throughout this paper provide a realistic approximation of real materials, and whether the optimal spectral characteristics we propose for efficient cells are achievable: narrow absorbance and emission line widths, with small stokes-shift. In order to test this, we have selected three model chromophores from the literature and performed calculations using their measured spectra to determine how each of these chromophores would perform in the multilayer energy-transfer solar cells we discuss in this paper. Specifically, we model multilayer stacks constructed from families of materials with properties similar to: (1) core-shell CdSe quantum dots (QDs), ${ }^{3}(2)$ anthrodithiophene triethylsilylethynyl (ADT-TES), ${ }^{4}$ and (3) boron subphthalocyanine chloride (SubPc) ${ }^{5}$ In each case certain assumptions must be made, as not all the required materials properties are reported in the literature. In the case of QDs, we estimate the dot density in a thin film at $\left(1\right.$ per $\left.1000 \mathrm{~nm}^{3}\right)$, consistent with the size of the dots and associated long-chain ligands in TEM images; ${ }^{3}$ for ADT-TES we estimate the peak thin-film absorption coefficient at $0.008 / \mathrm{nm}$, equivalent to that we have observed for TiOPc; for SubPc we assume a PL quantum yield of 50\%, consistent with the large Forster radius observed between SubPc and SubNc, ${ }^{6}$ and the large exciton diffusion lengths that have been reported. ${ }^{5}$

These materials exemplify, in one respect or another, many of the properties that we have predicted to be ideal for energy-transfer solar cells: narrow spectra, small stokes shifts, and high thin-film PL quantum yields.

Figure S8a shows a comparison of the short-circuit current density and power conversion efficiency predicted for optimized 5-layer structures made from each of these three classes of materials. The successive layers are defined by shifting the empirical spectra in energy such that the optical band gap of the lowest energy material is $1.5 \mathrm{eV}$. Each successive layer has a higher energy gap, suitable for directed energy transfer to the central "reaction center", and the energetic shift in the spectra from one material to the next is varied to find the optimal photon harvesting efficiency, as shown.

Perhaps surprisingly, given their high PL quantum yield and high molar extinction coefficients, CdSe quantum dots show very poor efficiency. This is mostly due to the relatively low density of dots that can be obtained in a thin film without quenching their luminescence, and the low absorption coefficient of the excitonic feature at the band edge. This translates into a very large effective stokes shift, even though the actual value is quite small. ADT-TES like materials fare much better, despite the lower potential PL quantum yield, but SubPc like materials are by far the best if a relatively high thin-film PL quantum yield can be assumed. Both ADT-TES and SubPc exhibit small stokes shifts, but the SubPc spectra are much narrower, which results in substantially improved efficiency. Indeed the predicted performance for SubPc like materials in this structure is equivalent to that of our optimized synthetic spectra.

Figure S8b shows how the performance of a multilayer composed of SubPc like materials would scale as a function of the number of layers and the thin film PL quantum yield, similar to the calculation for the synthetic spectra shown in S7a. For equivalent PL quantum yields, the SubPc-like materials achieve an efficiency that is $95 \%$ of that for our optimized synthetic spectra (14.7\% vs. $15.4 \%$ at $80 \%$ PLQY, 15 layers).

Figures S8c and d show the absorbance and quantum efficiency spectra for the 15-layer SubPc-like stack. Each layer is $10 \mathrm{~nm}$ thick. Again, as with the synthetic spectra of 
Figure S7, we find that substantial spectral overlap between neighboring materials is optimal.
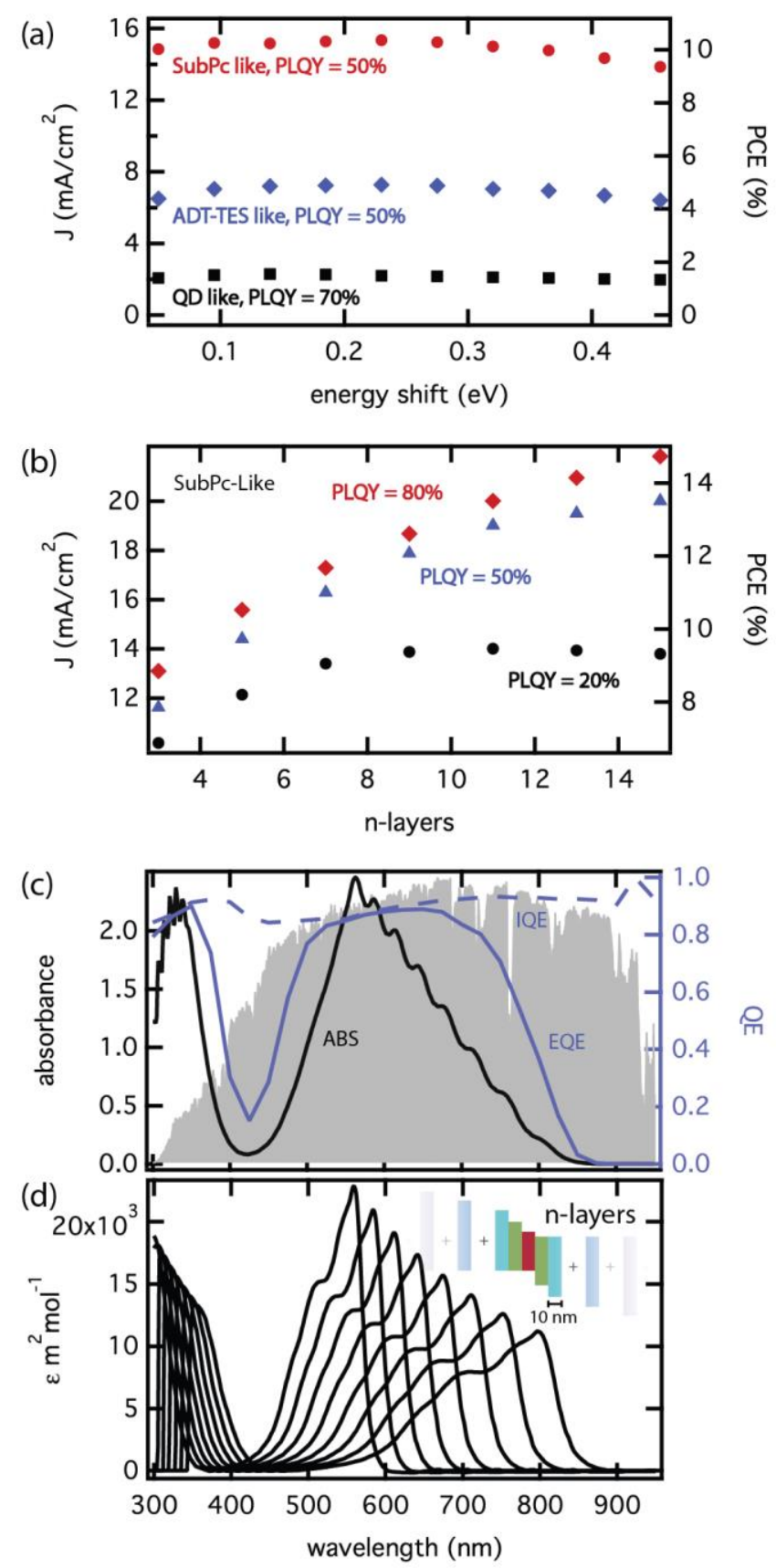

Figure S8) (a) comparison of current densities/efficiencies predicted for optimized 5layer structures formed from a set of empirically derived spectra for: core-shell CdSe quantum dots (black); anthrodithiophene triethylsilylethynyl (blue); and boron subphthalocyanine chloride (red). The $x$-axis is the shift in energy of the spectra of neighboring materials in the multilayer stack. Empirical absorption and emission spectra were used in each case. (b) The current density and efficiency predicted for multilayer structures with SubPc-like spectra in each layer. The $x$-axis is the number of layers in the stack, and results are plotted for three different photoluminescence quantum yield values: 
$80 \%$ (red); $50 \%$ (blue); and 20\% (black). (c) The total absorbance spectrum (black) and quantum efficiency spectra (blue) for the 15 layer structure of SubPc-like materials. The solar irradiance spectrum is plotted in grey. (d) Each individual absorption spectrum for the 15-layer stack of SubPc-like materials. Note that only 8-spectra are displayed, as layers on opposite sides of the stack have identical spectra.

\section{References:}

(1) Gierschner, J.; Mack, H.-G.; Lüer, L.; Oelkrug, D. Fluorescence and Absorption Spectra of Oligophenylenevinylenes: Vibronic Coupling, Band Shapes, and Solvatochromism. J. Chem. Phys. 2002, 116, 8596-8609.

(2) Shockley, W.; Queisser, H. J. Detailed Balance Limit of Efficiency of P-N Junction Solar Cells. J. Appl. Phys. 1961, 32, 510-519.

(3) Talapin, D. V.; Koeppe, R.; Götzinger, S.; Kornowski, A.; Lupton, J. M.; Rogach, A. L.; Benson, O.; Feldmann, J.; Weller, H. Highly Emissive Colloidal CdSe/CdS Heterostructures of Mixed Dimensionality. Nano Lett. 2003, 3, 1677-1681.

(4) Platt, A. D.; Day, J.; Subramanian, S.; Anthony, J. E.; Ostroverkhova, O. Optical, Fluorescent, and (Photo)Conductive Properties of High-Performance Functionalized Pentacene and Anthradithiophene Derivatives. J. Phys. Chem. C 2009, 113, 14006-14014.

(5) Gommans, H.; Schols, S.; Kadashchuk, A.; Heremans, P.; Meskers, S. C. J. Exciton Diffusion Length and Lifetime in Subphthalocyanine Films. J. Phys. Chem. C 2009, 113, 2974-2979.

(6) Rand, B. P.; Cheyns, D.; Verreet, B.; Empl, M. A.; Heremans, P.; Cnops, K. 8.4\% Efficient Fullerene-Free Organic Solar Cells Exploiting Long-Range Exciton Energy Transfer. Nat. Comm. 2014, 5, 3406. 